\title{
Standing Spin Waves in Ultrathin Magnetic Films: A Method to Test for Layer-Dependent Exchange Coupling
}

\author{
J. Rajeswari, ${ }^{1,2, *}$ H. Ibach, ${ }^{3,2}$ and C. M. Schneider ${ }^{1,2}$ \\ ${ }^{1}$ Peter Grünberg Institut (PGI-6), Forschungszentrum Jülich, 52425 Jülich, Germany \\ ${ }^{2} J$ ülich Aachen Research Alliance, 52425 Jülich, Germany \\ ${ }^{3}$ Peter Grünberg Institut (PGI-3), Forschungszentrum Jülich, 52425 Jülich, Germany
}

(Received 22 October 2013; published 26 March 2014)

\begin{abstract}
We introduce a method to test theoretical models for the layer dependence of exchange coupling constants in ultrathin magnetic films. The method is based on the observation of high-energy and highmomentum standing spin wave modes using high-resolution electron energy loss spectroscopy. Experimental data are presented for 5-8 layers of fcc cobalt deposited on $\mathrm{Cu}(100)$. The power of the method is illustrated by comparison to two theoretical studies predicting rather different results concerning the ratio of the interlayer and intralayer exchange coupling constants near the surface. Only the theory with a large interlayer coupling shows sufficient energy spreading in the layer dependence of the dispersion curves to match the experimental data. We furthermore discuss the reason for the surprising success of the simple nearest-neighbor Heisenberg model with a single exchange constant matched to experiment.
\end{abstract}

DOI: 10.1103/PhysRevLett.112.127202

PACS numbers: 75.30.Ds

The magnetic properties of thin films present an active field in contemporary research, partly because of the importance of magnetic films in information technology, partly because of the scientific challenges involved in our fundamental understanding of magnetism. For several decades, the interplay between finite dimensions, lowered symmetry and the crystallography of thin epitaxial films and the magnetic properties such as the orientation of the easy axis, the magnetization hysteresis, and the nature of domain walls have been studied [1-5]. Motivated by the emergence of atomic scale probes, the focus has lately shifted to the magnetic properties of individual atoms located at the surface or at the interface and specific magnetic ordering phenomena that appear there [6-11]. The two most fundamental properties of atoms determining the magnetic order are the magnetic moments of atoms and the exchange coupling between them.

Concerning the magnetic moments of atoms in ferromagnetic films, theory and experiment have painted a clear picture. Theoretical studies predict an enhancement of the magnetic moments at surfaces and a reduction in the layer adjacent to nonferromagnetic substrates [12-15]. Moments inside an adjacent nonferromagnetic substrate may be enlarged [16]. Experimental studies on magnetic moments of atoms using x-ray magnetic circular dichroism [17-19] confirm these conclusions.

With regard to layer dependence of exchange coupling constants between atoms, the situation is less clear. Theoretical papers have come to contradictory conclusions. Experimental methods to probe for the layer specificity of the exchange coupling are hitherto unknown. For the specific case of fcc cobalt films deposited on $\mathrm{Cu}(100)$, e.g., Costa et al. found an enhancement of the surface intralayer exchange coupling by $90 \%$ and by $40 \%$ in the layer next to the substrate interface [20]. The interlayer exchange coupling between the surface layer and the next layer underneath was also found to be enhanced, but much less so. Layers second to the surface or the interface were less affected. A very different result was recently reported by Bergqvist et al. [21]: According to their calculations the intralayer exchange coupling at the surface is higher by about $50 \%$, however, reduced to about $60 \%$ in the next layer below the surface. The interlayer coupling between the surface layer and the second layer, however, was found to be almost a factor of 2 larger than the intralayer constant in the interior of the film.

In this Letter, we show that the considerable discrepancy between the two theories concerning the relative strength of the interlayer coupling can be probed experimentally by studying high-energy and high momentum surface spin waves [22-25] in combination with the recently discovered lowest standing spin waves [26] in ultrathin ferromagnetic films. To avoid misunderstandings we note that the frequency regime of interest here lies between 5-25 THz, far above the range of magnetostatic spin waves. Frequency and dispersion of these high frequency spin waves are entirely determined by exchange coupling.

To illustrate our method we consider a ferromagnetic slab. For wave vectors parallel to the surface of the slab $q_{\|}$ which are large compared to the inverse of the thickness $1 / d$ each of the two surfaces of the slab carries a surface spin wave mode [27]. Because of the mirror symmetry around the center of the film, the total eigensolution decomposes into a symmetric and antisymmetric combination of the two surface modes. For smaller wave vectors ( $q_{\|}$comparable to $1 / d$ ), the symmetric combination of the 
two surface modes becomes a uniform mode in which all spins precess in phase. This mode was observed in earlier studies [22,26]. As its frequency is rather insensitive to the thickness of the slab and close to the frequency of the surface mode of a semi-infinite bulk it is generally addressed as the "surface mode." We keep this notation in the remainder of the Letter. The frequency of the surface mode is a measure of the average exchange coupling in the film. The antisymmetric combination of the two surface modes has a node in the amplitude at or near the center of the film. Because of the node, the antisymmetric combination of the two surface modes can be considered as the lowest standing mode. Higher standing modes possess more nodes. The total number of all modes equals the number of layers. For details, see the Supplemental Material [28].

So far, higher standing modes have not been observed in electron spectroscopy because of their strong damping. The value of studying the lowest standing mode in addition to the surface mode rests with the sensitivity of that mode to the thickness of the slab. The presence of the node causes the amplitudes to vary more strongly from layer to layer compared to the symmetric mode with no node. Because of the exchange coupling between the layers, the frequency of the standing mode is therefore always higher than the frequency of the surface mode. More importantly in this context, the lowest standing mode is more sensitive to the exchange coupling at the surface and the interface, in particular, to the interlayer exchange coupling. Therefore, by observing the lowest standing spin wave mode as a function of film thickness and by comparing the frequencies of the standing mode and the surface mode for different film thicknesses as a function of $q_{\|}$, one obtains information on the layer dependence of the exchange coupling. In particular, theoretical models can be tested.

Standing spin wave modes have been observed previously using scanning tunneling spectroscopy [29]. However, because of the atomic localization of the probe, the parallel momentum of the spin wave excitation is presumably not well defined in scanning tunneling spectroscopy. Furthermore, the most significant data in those experiments stem from thicker films. The standing mode frequencies were therefore dominated by the bulk properties of the film material [29]. Here, we employ inelastic electron scattering by which technique spin waves are observable even on films with a thickness of only a few layers. In such scattering experiments the wave vector of the spin waves parallel to the surface $q_{\|}$is defined via the wave vector conservation

$$
\Delta K_{\|}=k^{(\mathrm{i})} \sin \theta^{(\mathrm{i})}-k^{(\mathrm{s})} \sin \theta^{(\mathrm{s})}=-q_{\|},
$$

in which $k^{(\mathrm{s})}, \theta^{(\mathrm{s})}$ and $k^{(\mathrm{i})}, \theta^{(\mathrm{i})}$, denote the wave vectors and polar angles of scattered and incident electrons, respectively.

Evidence for a standing spin wave in high-resolution energy loss spectra was demonstrated in a recent publication of ours for an eight-atom layers (8 ML) thick Co film deposited on $\mathrm{Cu}(100)$ [26]. Presently, our spectrometer is technically further improved so that standing spin wave modes are observed with several hundred counts per second and an energy resolution of $7 \mathrm{meV}$. In this Letter, we report on the dispersion of the lowest standing modes for five, six, seven, and eight-monolayer thick fcc cobalt films deposited on $\mathrm{Cu}(100)$ together with the dispersion of the surface modes of such films. The experimental data are analyzed within the framework of the Heisenberg model using the nearest and next-nearest neighbor exchange constants of Costa et al. [20] and Bergqvist et al. [21]. We find that neither theory fits the data per se. The model of Bergqvist et al., however, provides a good fit when all coupling constants are uniformly scaled down to $85 \%$. The model of Costa $e t a l$., on the other hand, does not produce a large enough spreading in the energies of the lowest standing modes as a function of the cobalt layer thickness due to the comparatively lower value of the interlayer coupling between the surface and the next layer.

Our samples are prepared according to the procedure described in Ref. [26]. All spin wave spectra were recorded with a scattering angle of $90^{\circ}$. The momentum transfer parallel to the surface is adjusted by the rotation of the sample manipulator around a vertical axis in the surface plane. The wave vector transfer is along the $\langle 110\rangle(\bar{\Gamma} \bar{X})$ direction. Figure 1 shows a series of spin wave spectra

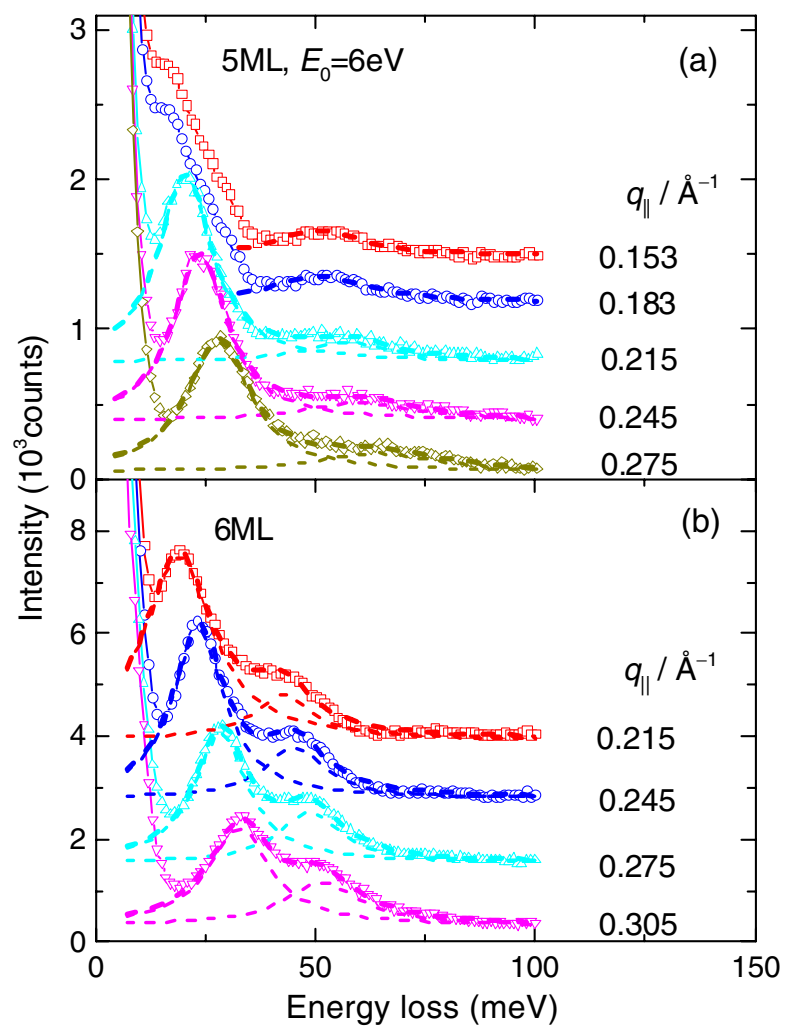

FIG. 1 (color online). Series of spin wave spectra of the (a) 5 and (b) $6 \mathrm{ML} \mathrm{Co} / \mathrm{Cu}(100)$ system as function of wave vector $q_{\|}$. Two modes of spin waves are clearly visible. 
measured on 5 and 6 ML films of fcc cobalt on $\mathrm{Cu}(100)$ ranging from $q_{\|}=0.153$ to $0.305 \AA^{-1}$. The FWHM of the diffuse elastic peak is about $7 \mathrm{meV}$. The impact energy is chosen as $E_{0}=6 \mathrm{eV}$, where the cross section for spin wave excitation has a maximum [30-32]. For each wave vector, two peaks are clearly seen. The pronounced peak at lower energy corresponds to the excitation of the surface spin wave, whereas the higher energy peak is the first standing spin wave mode. The dashed lines represent fits of the spin wave spectra by two Lorentzians from which the peak energies are determined.

We note in passing that the strong signature of the standing modes in the spectra is at variance with the conclusions of Taroni et al. [33]. The reason for the discrepancy is that Taroni et al. erroneously assumed that all layers inside the film contribute to the scattering amplitude with the same weight, as would be the case in inelastic neutron scattering. This leads to a cancellation of the scattering amplitude because of the $180^{\circ}$ phase shift between the spin wave amplitudes in surface and the interface layer. In electron scattering, only the top two layers contribute significantly to the scattering amplitude and the surface mode and first standing mode have about the same amplitude there (see, e.g., [20]). The same argument could be brought forward for the higher standing modes. However, those modes, because of their higher energy, are too strongly Landau damped to be observable (see Fig. 12 of [26]).

Figure 2 shows the dispersion of the modes in the relevant range of small wave vectors for films consisting of 5-8 layers. We examined also four-layer films. While the surface mode is clearly seen for those films (see also [34]) the standing mode was too broad to be resolved from the continuous background. The open and solid symbols represent the data points for the surface mode and the standing mode, respectively. Within the limits of error, the dispersion of the surface mode is independent of film thickness whereas the dispersion of the standing mode varies strongly with the thickness.

The experimental data are compared to dispersion curves generated within the Heisenberg model using previously published nearest and next-nearest exchange coupling constants $[20,21]$. While the two theories employ different schemes to describe the ground state properties of the system, in both cases the published coupling constants are obtained assuming that the itinerant electron system responds adiabatically to the motion of localized spins. The description of the spin dynamics by using these coupling constants in a Heisenberg model is in general not justified in the case of $3 d$ metals, as was demonstrated clearly in Ref. [20]. The deviation between the Heisenberg model and the full dynamic response function is particularly large for larger wave vectors. However, here we are interested in the low $q$ limit where the Landau damping due to Stoner excitations is small. The Heisenberg model is therefore considered a reasonable approach. We emphasize that the

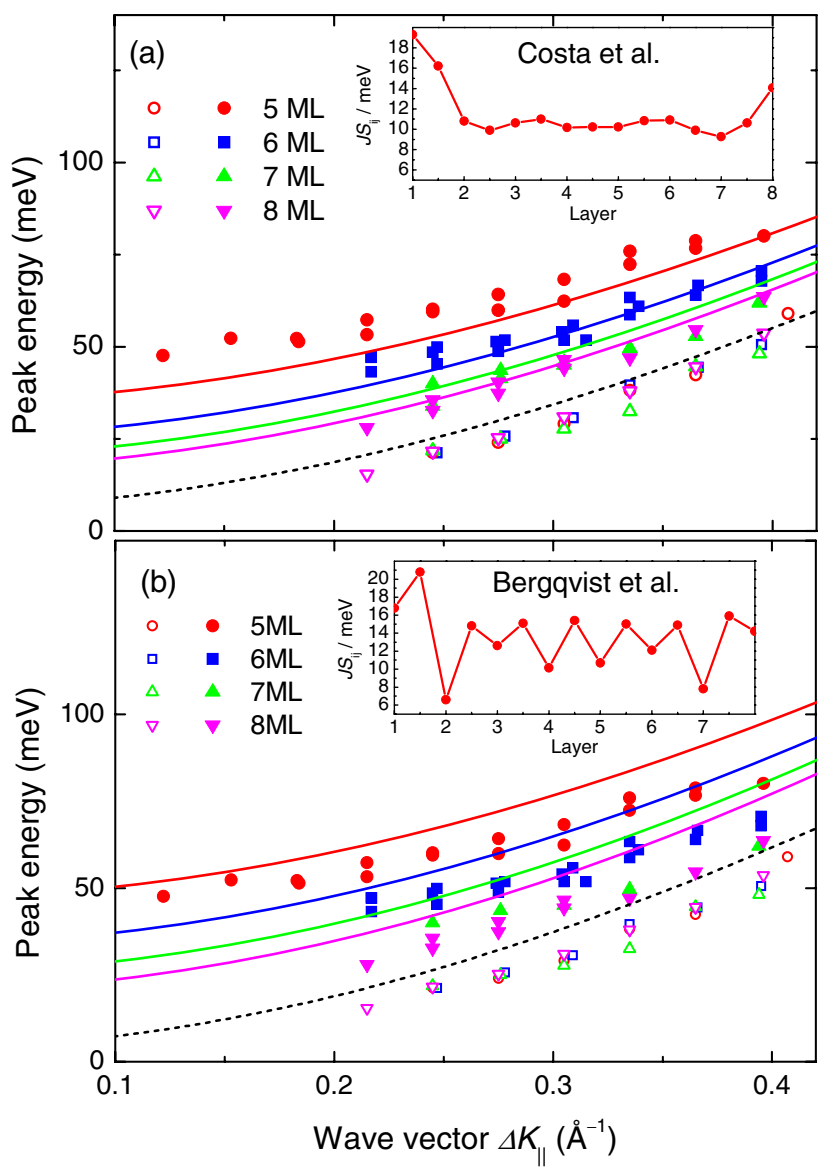

FIG. 2 (color online). Dispersion of the surface mode (open symbols) and the standing mode (solid symbols) as function of cobalt film thickness. The red circles, blue squares, green up triangles, and magenta down triangles are for the five, six, seven, and eight-layer system, respectively. The solid and dashed lines are the standing modes and the surface modes, respectively, calculated with the coupling parameters of (a) Costa et al. [20] and (b) Bergqvist et al. [21]. The insets display the nearestneighbor intralayer and interlayer exchange-coupling constants at integer and half-integer positions, respectively.

objective of our study is not to prove a particular theory "wrong" or "right," but rather to show that standing waves have the potential to distinguish between different theoretical approaches.

The solid and dashed lines in Fig. 2 show the dispersion of the standing modes and the surface modes, respectively, as calculated with the nearest and next-nearest exchange coupling constants of (a) Costa et al. [20] and (b) Bergqvist et al. [21]. In both theoretical papers, the parameters are tabulated for an eight-layer film. In order to calculate the dispersion for 5-7 layer films we make use of the fact that the coupling constants are nearly constant from the 3rd to the 5th layer inside the film. The dispersion for the 5-7 layer films is therefore calculated by first replacing the layer-dependent constants of the 3rd to 5th layer by their 
average and, second, by removing those layers sequentially from the film to realize 7-5 layer films.

As seen from Fig. 2, neither set of exchange coupling constants yields a satisfactory fit to the experimental data. The disagreement is, however, of a different nature in the two cases. The dispersion curves according to Costa et al. [Fig. 2(a)] have too small energy spreading in the layer dependence of the dispersion curves. A uniform up- or downscaling of the coupling constants would not lead to a better agreement with experiment. A variation of merely the increase of the exchange coupling near the surface or interface likewise does not lead to a satisfactory agreement with experiment. While the dispersion curves obtained for the parameter set of Bergqvist et al. also do not fit the experiment they can be brought to agreement by a uniform downscaling of all parameters. This is illustrated in Fig. 3(a) where the dispersion curves are shown with all parameters reduced to $85 \%$ of their published value. Hence, the theory of Bergqvist does reproduce the experimentally required energy spreading in the layer dependence of the dispersion curves. The reason for the success of the theory and the failure of the other with respect to the energy spreading is the different interlayer coupling near the surface in the two theoretical studies. Bergqvist et al. find a large interlayer coupling near the surface, larger than the intralayer coupling (see insets in Fig. 2). Costa et al., on the other hand, have the interlayer coupling near the surface smaller than the intralayer coupling. Model calculations with varied parameters as well as the reasoning brought forward in the introduction show that the different ratio of the interlayer and intralayer couplings in the two theories is the key for the different quality of the (dis)agreement with experiment. Model studies further show that the small oscillations between the interlayer and intralayer couplings in the interior of the films in case of Bergqvist et al. are of no significant consequence for the result, given the present precision of the experimental data.

Amusingly, the agreement between experiment and the theory of Bergqvist et al. even after downscaling is not better than with the simplest of all models, the nearest neighbor Heisenberg model with a uniform exchange coupling constant if this coupling constant is chosen to be $J S=15 \mathrm{meV}$ [Fig. 3(b)]. The simple model even fits the dispersion of the surface mode up to the zone boundary with the same exchange coupling [22] as good as the downscaled theory of Bergqvist et al. Model calculations show that the relatively large spreading of the energies in the simple nearest-neighbor model (despite the interlayer coupling being equal to the intralayer coupling) is owed to the neglect of second and higher neighbor interactions in combination with a relatively high nearest-neighbor coupling constant (higher than obtained in theory) chosen to match the experiments. For models with a realistic range of the exchange coupling, we can safely exclude those that have the interlayer coupling near the surface smaller than the intralayer coupling.

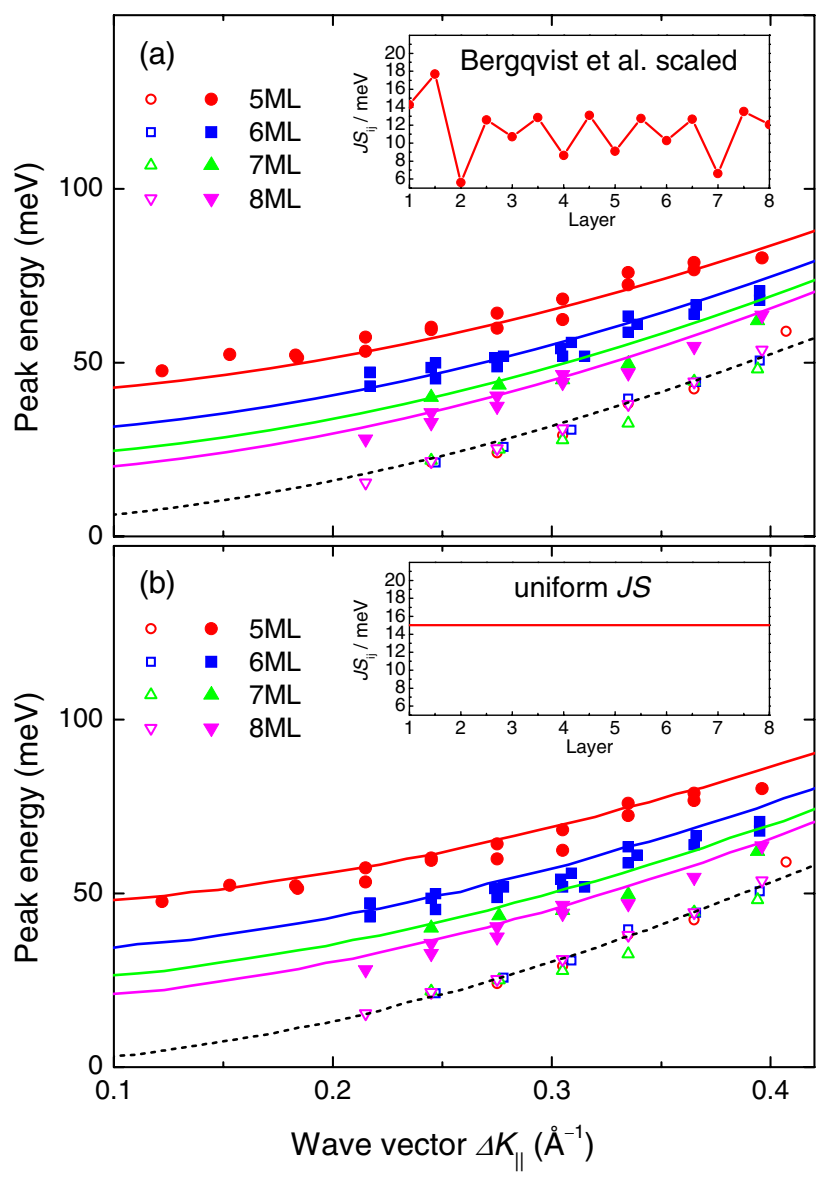

FIG. 3 (color online). Experimental data as in Fig. 2. The solid and dashed lines are the standing modes and the surface modes, respectively, calculated with the coupling parameters of (a) Bergqvist et al. reduced to $85 \%$ [21]. Surprisingly, the simplest of all models, the nearest neighbor Heisenberg model with a uniform exchange coupling constant of $J S=15 \mathrm{meV}$ fits the data just as well (b) (see text for discussion). The insets display the nearestneighbor intralayer and interlayer exchange-coupling constants at integer and half-integer positions, respectively.

A possible reason for the qualitative difference between the theoretical models of Costa et al. and Bergqvist et al. is that only in the latter case interlayer distances were relaxed to achieve a minimum of the total energy. Given the sensitivity of the exchange coupling on the interatomic distances we consider this to be the likely cause for the qualitative difference between the two theories. In summary, we have shown that a careful study of the dispersion of the two types of spin wave modes as a function of the layer thickness has the potential to discriminate between theoretical models and to point out key requirements for a theory to match experiment.

The authors have benefited from enlightening discussions with A. T. Costa, S. Lounis, C. Etz, and L. Bergqvist. The able technical support of B. Küpper is gratefully acknowledged. 
*r.jayaraman@fz-juelich.de

[1] C. Schneider and J. Kirschner, in Electronic Structure, Handbook of Surface Science Vol. 2, edited by K. Horn and M. Scheffler (North-Holland, Amsterdam, 2000), p. 511.

[2] C. A. F. Vaz, J. A. C. Bland, and G. Lauhoff, Rep. Prog. Phys. 71, 056501 (2008).

[3] D. L. Mills, in Ultrathin Magnetic Structures I, edited by J. Bland and B. Heinrich (Springer, Berlin, Heidelberg, 2005) p. 91.

[4] M. Farle, W. Platow, A. N. Anisimov, P. Poulopoulos, and K. Baberschke, Phys. Rev. B 56, 5100 (1997).

[5] G. Chen, J. Zhu, A. Quesada, J. Li, A. T. N'Diaye, Y. Huo, T. P. Ma, Y. Chen, H. Y. Kwon, C. Won, Z. Q. Qiu, A. K. Schmid, and Y. Z. Wu, Phys. Rev. Lett. 110, 177204 (2013).

[6] M. Bode and R. Wiesendanger, in Magnetic Microscopy of Nanostructures, NanoScience and Technology, edited by H. Hopster and H. Oepen (Springer, Berlin, Heidelberg, 2005) p. 203.

[7] M. Bode, M. Heide, K. von Bergmann, P. Ferriani, S. Heinze, G. Bihlmayer, A. Kubetzka, O. Pietzsch, S. Blügel, and R. Wiesendanger, Nature (London) 447, 190 (2007).

[8] T. Balashov, T. Schuh, A. F. Takács, A. Ernst, S. Ostanin, J. Henk, I. Mertig, P. Bruno, T. Miyamachi, S. Suga, and W. Wulfhekel, Phys. Rev. Lett. 102, 257203 (2009).

[9] S. Heinze, K. von Bergmann, M. Menzel, J. Brede, A. Kubetzka, R. Wiesendanger, G. Bihlmayer, and S. Blügel, Nat. Phys. 7, 713 (2011).

[10] F. Meier, S. Lounis, J. Wiebe, L. Zhou, S. Heers, P. Mavropoulos, P. H. Dederichs, S. Blügel, and R. Wiesendanger, Phys. Rev. B 83, 075407 (2011).

[11] A. A. Khajetoorians, S. Lounis, B. Chilian, A. T. Costa, L. Zhou, D. L. Mills, J. Wiebe, and R. Wiesendanger, Phys. Rev. Lett. 106, 037205 (2011).

[12] A. J. Freeman and R.-q. Wu, J. Magn. Magn. Mater. 104-107, 1 (1992).

[13] A. M. N. Niklasson, B. Johansson, and H. L. Skriver, Phys. Rev. B 59, 6373 (1999).

[14] C. Vaz, G. Lauhoff, J. Bland, S. Langridge, D. Bucknall, J. Penfold, J. Clarke, S. Halder, and B. Tanner, J. Magn. Magn. Mater. 313, 89 (2007).

[15] Z. Yang, V. Gavrilenko, and R. Wu, Surf. Sci. 447, 212 (2000).

[16] D. L. R. Santos, P. Venezuela, R. B. Muniz, and A. T. Costa, Phys. Rev. B 88, 054423 (2013).
[17] M. Martins, P. Gastelois, R. Landers, andW. Macedo, J. Magn. Magn. Mater. 310, 2274 (2007).

[18] A. Ney, P. Poulopoulos, and K. Baberschke, Europhys. Lett. 54, 820 (2001).

[19] A. Ney, A. Scherz, P. Poulopoulos, K. Lenz, H. Wende, K. Baberschke, F. Wilhelm, and N. B. Brookes, Phys. Rev. B 65, 024411 (2001).

[20] A. T. Costa, R. B. Muniz, and D. L. Mills, Phys. Rev. B 70, 054406 (2004).

[21] L. Bergqvist, A. Taroni, A. Bergman, C. Etz, and O. Eriksson, Phys. Rev. B 87, 144401 (2013).

[22] R. Vollmer, M. Etzkorn, P. S. A. Kumar, H. Ibach, and J. Kirschner, Phys. Rev. Lett. 91, 147201 (2003).

[23] J. Prokop, W. X. Tang, Y. Zhang, I. Tudosa, T. R. F. Peixoto, K. Zakeri, and J. Kirschner, Phys. Rev. Lett. 102, 177206 (2009).

[24] J. Rajeswari, H. Ibach, and C. M. Schneider, Europhys. Lett. 101, 17003 (2013).

[25] J. Rajeswari, H. Ibach, and C. M. Schneider, Phys. Rev. B 87, 235415 (2013).

[26] J. Rajeswari, H. Ibach, C. M. Schneider, A. T. Costa, D. L. R. Santos, and D. L. Mills, Phys. Rev. B 86, 165436 (2012).

[27] D. L. Mills, Surface Excitations, edited by V. Agranovich and R. Loudon, Modern Problems in Condensed Matter Sciences (North-Holland, Amsterdam, 1984).

[28] See Supplemental Material at http://link.aps.org/ supplemental/10.1103/PhysRevLett.112.127202 for detailed information.

[29] C. L. Gao, A. Ernst, G. Fischer, W. Hergert, P. Bruno, W. Wulfhekel, and J. Kirschner, Phys. Rev. Lett. 101, 167201 (2008).

[30] J. Rajeswari, E. Michel, H. Ibach, and C. M. Schneider, Phys. Rev. B 89, 075438 (2014).

[31] M. Etzkorn, Ph.D. thesis, Martin-Luther-Universität HalleWittenberg, 2005, http://nbn-resolving.de/urn/resolver.pl? urn=nbn \%3Ade\%3Agbv\%3A3-000008590.

[32] R. Vollmer, M. Etzkorn, P. Kumar, H. Ibach, and J. Kirschner, Thin Solid Films 464-465, 42 (2004).

[33] A. Taroni, A. Bergman, L. Bergqvist, J. Hellsvik, and O. Eriksson, Phys. Rev. Lett. 107, 037202 (2011).

[34] M. Etzkorn, P. A. Kumar, R. Vollmer, H. Ibach, and J. Kirschner, Surf. Sci. 566-568, 241 (2004). 